\title{
Degenerate Poly-Lah-Bell Polynomials and Numbers
}

\author{
Taekyun Kim $\mathbb{D}^{1}$ and Hye Kyung Kim $\mathbb{D}^{2}$ \\ ${ }^{1}$ Department of Mathematics, Kwangwoon University, Seoul 139-701, Republic of Korea \\ ${ }^{2}$ Department of Mathematics Education, Daegu Catholic University, Gyeongsan 38430, Republic of Korea
}

Correspondence should be addressed to Hye Kyung Kim; hkkim@cu.ac.kr

Received 29 June 2021; Accepted 15 January 2022; Published 25 February 2022

Academic Editor: Ali Jaballah

Copyright (c) 2022 Taekyun Kim and Hye Kyung Kim. This is an open access article distributed under the Creative Commons Attribution License, which permits unrestricted use, distribution, and reproduction in any medium, provided the original work is properly cited.

\begin{abstract}
Many mathematicians studied "poly" as a generalization of the well-known special polynomials such as Bernoulli polynomials, Euler polynomials, Cauchy polynomials, and Genocchi polynomials. In this paper, we define the degenerate poly-Lah-Bell polynomials arising from the degenerate polyexponential functions which are reduced to degenerate Lah-Bell polynomials when $k=1$. In particular, we call these polynomials the "poly-Lah-Bell polynomials" when $\lambda \longrightarrow 0$. We give their explicit expression, Dobinski-like formulas, and recurrence relation. In addition, we obtain various algebraic identities including Lah numbers, the degenerate Stirling numbers of the first and second kind, the degenerate poly-Bell polynomials, the degenerate poly-Bernoulli numbers, and the degenerate poly-Genocchi numbers.
\end{abstract}

\section{Introduction}

Many mathematicians have studied "poly" for the wellknown special polynomials such as poly-Bernoulli polynomials, poly-Euler polynomials, poly-Bell polynomials, and poly-Genocchi polynomials [1-10]. Also, a lot of research has been done on degenerate versions of various special polynomials and numbers, such as Stirling numbers, Bernoulli polynomials, Euler polynomials, Genocchi polynomials, and Bell polynomials, which has given mathematicians a renewed interest in various degenerate polynomials and numbers $[1,3,4,6,7,9,11]$. Recently, we introduced degenerate poly-Bell polynomials as one of the generalizations of ordinary Bell polynomials [4]. In relation to this, in this paper, we define the degenerate polyLah-Bell polynomials by means of the degenerate polyexponential functions called the "poly-Lah-Bell polynomial"s when $\lambda \longrightarrow 0$. These polynomials are reduced to degenerate Lah-Bell polynomials when $k=1$. In particular, they are reduced to degenerate Lah-Bell polynomials if $k=1$. We derive their explicit expression, Dobinski-like formulas, recurrence relation, and various algebraic identities including Lah numbers, the degenerate Stirling numbers of the first and second kind, the degenerate poly-
Bell polynomials, the degenerate poly-Bernoulli numbers, and the degenerate poly-Genocchi numbers.

The Lah numbers, which was studied by Lah in 1955 [12], have many other interesting applications in analysis and combinatorics (see [13-15]).

The unsigned Lah number $L(n, k)$ counts the number of ways to partition the set $\{1,2, \ldots, n\}$ into $k$ nonempty linearly ordered subsets, and (see $[3,12,13])$

$$
\mathbf{L}(n, k)=\left(\begin{array}{c}
n-1 \\
k-1
\end{array}\right) \frac{n !}{k !} \quad k \geq 0 .
$$

From (1) (see [13, 14]), the generating function of $\mathbf{L}(n, k)$ is given by

$$
\frac{1}{k !}\left(\frac{t}{1-t}\right)^{k}=\sum_{n=k}^{\infty} \mathrm{L}(n, k) \frac{t^{n}}{n !}, \quad k \geq 0 .
$$

Kim-Kim [14] introduced the Lah-Bell polynomials $B_{n}^{L}(x)$ given by

$$
e^{x(t /(1-t))}=e^{x((1 /(1-t))-1)}=\sum_{n=0}^{\infty} B_{n}^{L}(x) \frac{t^{n}}{n !} .
$$


When $x=1$, Lah-Bell numbers are given by $e^{(1 /(1-t))-1}=\sum_{n=0}^{\infty} B_{n}^{L}\left(t^{n} / n !\right)$.

It was known that (see [13])

$$
(1-t)^{-m}=\sum_{l=0}^{\infty}\left(\begin{array}{c}
-m \\
l
\end{array}\right)(-1)^{l} t^{l}=\sum_{l=0}^{\infty}\langle m\rangle_{l} \frac{t^{l}}{l !},
$$

where $\langle x\rangle_{0}=1$ and $\langle x\rangle_{n}=x(x+1) \cdots(x+n-1)$.

For $\lambda \in \mathbb{R}$, the degenerate exponential function is defined by (see $[1,3,4,6,7,11])$

$$
\begin{aligned}
& e_{\lambda}^{x}(t)=(1+\lambda t)^{x / \lambda} \\
& e_{\lambda}(t)=\sum_{n=0}^{\infty}(1)_{n, \lambda} \frac{t^{n}}{n !}
\end{aligned}
$$

where $\quad(x)_{0, \lambda}=1 \quad$ and $\quad(x)_{n, \lambda}=x(x-\lambda)(x-2 \lambda) \cdots$ $(x-(n-1) \lambda)$.

In this paper, the degenerate Lah-Bell polynomials are given by (see [14])

$$
e_{\lambda}\left(x\left(\frac{t}{1-t}\right)\right)=\sum_{n=0}^{\infty} B_{n, \lambda}^{L} \frac{t^{n}}{n !} .
$$

For an integer $k$, the polylogarithm function is defined by (see $[6,16])$

$$
L i_{k}(x)=\sum_{m=1}^{\infty} \frac{x^{m}}{m^{k}} .
$$

When $k=1, L i_{1}(x)=-\log (1-t)$.

Recently, the polyexponential functions introduced by Kim-Kim are given by (see [8])

$$
E i_{k}(x)=\sum_{n=1}^{\infty} \frac{x^{n}}{(n-1) ! n^{k}}, \quad k \in \mathbb{Z} .
$$

By (8), we see that $E i_{1}(x)=e^{x}-1$.

The degenerate polyexponential functions also are given by (see $[6,8])$

$$
E i_{k, \lambda}(x)=\sum_{n=1}^{\infty} \frac{(1)_{n, \lambda} x^{n}}{(n-1) ! n^{k}}, \quad k \in \mathbb{Z} .
$$

We note that $E i_{1, \lambda}(x)=e_{\lambda}(x)-1$.

The degenerate poly-Bernoulli polynomials are given by (see [4])

$$
\frac{E i_{k, \lambda}\left(\log _{\lambda}(1+t)\right)}{e_{\lambda}(t)-1} e_{\lambda}^{x}(t)=\sum_{n=0}^{\infty} \beta_{n, \lambda}^{(k)}(x) \frac{t^{n}}{n !} .
$$

When $x=0, \beta_{n, \lambda}^{(k)}=\beta_{n, \lambda}^{(k)}(0)$ are called the degenerate poly-Bernoulli numbers.

The degenerate poly-Genocchi polynomials are given by (see [3])

$$
\frac{2 E i_{k, \lambda}\left(\log _{\lambda}(1+t)\right)}{e_{\lambda}(t)+1} e_{\lambda}^{x}(t)=\sum_{n=0}^{\infty} \mathbb{G}_{n, \lambda}^{(k)}(x) \frac{t^{n}}{n !} .
$$

When $x=0, \mathbb{G}_{n, \lambda}^{(k)}=\mathbb{G}_{n, \lambda}^{(k)}(0)$ are called the degenerate poly-Genocchi numbers.
The degenerate Stirling numbers of the second and fist kind are given by (see $[3,4])$

$$
\begin{aligned}
\frac{1}{k !}\left(e_{\lambda}(t)-1\right)^{k} & =\sum_{n=k}^{\infty} S_{2, \lambda}(n, k) \frac{t^{n}}{n !}, \\
\frac{1}{k !}\left(\log _{\lambda}(1+t)\right)^{k} & =\sum_{n=k}^{\infty} S_{1, \lambda}(n, k) \frac{t^{n}}{n !}, \quad k \geq 0,
\end{aligned}
$$

respectively $($ see $[4,9])$. where $\log _{\lambda}\left(e_{\lambda}(t)\right)=e_{\lambda}\left(\log _{\lambda}(t)\right)=t$ and $\log _{\lambda}(t)=(1 / \lambda)\left(t^{\lambda}-1\right)$.

Recently, for $k \in \mathbb{Z}$, we introduced the degenerate polyBell polynomials $b e l_{n, \lambda}^{(k)}(x)$ given by (see [4])

$$
E i_{k, \lambda}\left(x\left(e_{\lambda}(t)-1\right)\right)+1=\sum_{n=0}^{\infty} b e l_{n, \lambda}^{(k)}(x) \frac{t^{n}}{n !},
$$

and $b e l_{0, \lambda}^{(k)}(x)=1$.

When $x=1, b e l_{n, \lambda}^{(k)}=b e l_{n, \lambda}^{(k)}(1)$ are called the degenerate poly-Bell numbers.

When $\lambda \longrightarrow 0, \operatorname{bel}_{n}^{(k)}(x)$ are called the poly-Bell polynomials.

\section{Degenerate Poly-Lah-Bell Polynomials and Numbers}

In this section, we introduce the degenerate poly-Lah-Bell polynomials by using of the degenerate polyexponential functions and give explicit expression and various noble identities involving those polynomials.

For $k \in \mathbb{Z}$, we define the degenerate poly-Lah-Bell polynomials $L b_{n, \lambda}^{(k)}(x)$, which are arising from degenerate polyexponential functions to be

$$
E i_{k, \lambda}\left(\frac{t}{1-t} x\right)+1=\sum_{n=0}^{\infty} L b_{n, \lambda}^{(k)}(x) \frac{t^{n}}{n !},
$$

and $L b_{0, \lambda}^{(k)}(x)=1$.

When $x=1, L b_{n, \lambda}^{(k)}=L b_{n, \lambda}^{(k)}(1)$ are called degenerate polyLah-Bell numbers.

When $\lambda \longrightarrow 0, L b_{n}^{(k)}(x)$ are called the poly-Lah-Bell polynomials.

When $k=1$, from (6), we have

$$
\begin{aligned}
E i_{1, \lambda}\left(\frac{t}{1-t} x\right)+1 & =1+\sum_{n=1}^{\infty} \frac{(1)_{n, \lambda}(t /(1-t) x)^{n}}{(n-1) ! n} \\
& =e_{\lambda}\left(\frac{t}{1-t} x\right)=\sum_{n=0}^{\infty} B_{n, \lambda}^{L}(x) .
\end{aligned}
$$

Combining (15) and (16), we obtain

$$
L b_{1, n, \lambda}^{(1)}(x)=B_{n, \lambda}^{L}(x) .
$$

Theorem 1. For $k \in \mathbb{Z}$, 


$$
\begin{aligned}
& L b_{0, \lambda}^{(k)}(x)=1 \\
& L b_{n, \lambda}^{(k)}(x)=\sum_{s=1}^{n} \frac{(1)_{s, \lambda}}{s^{k-1}} L(n, s) x^{s}, \quad n \geq 1 .
\end{aligned}
$$

Proof. From (2), (9), and (15),

$$
\begin{aligned}
E i_{k, \lambda}\left(\frac{t}{1-t} x\right) & =\sum_{s=1}^{\infty} \frac{(1)_{s, \lambda}}{(s-1) ! s^{k}}\left(\frac{t}{1-t} x\right)^{s} \\
& =\sum_{s=1}^{\infty} \frac{(1)_{s, \lambda}}{s^{k-1}} x^{s} \frac{1}{s !}\left(\frac{t}{1-t}\right)^{s} \\
& =\sum_{s=1}^{\infty} \frac{(1)_{s, \lambda}}{s^{k-1}} x^{s} \sum_{n=s}^{\infty} L(n, s) \frac{t^{n}}{n !} \\
& =\sum_{n=1}^{\infty}\left(\sum_{s=1}^{n} \frac{(1)_{s, \lambda}}{s^{k-1}} L(n, s) x^{s}\right) \frac{t^{n}}{n !}
\end{aligned}
$$

By (15) and (19), we have the desired result.

Theorem 2 (Dobinski-like formulas). For $k \in \mathbb{Z}$ and $n \geq 1$,

$$
L b_{n, \lambda}^{(k)}(x)=\sum_{m=1}^{\infty} \sum_{j=0}^{m}\left(\begin{array}{c}
m \\
j
\end{array}\right) \frac{(1)_{m, \lambda}(-1)^{m-j}\langle j\rangle_{n}}{(m-1) ! m^{k}} x^{m} .
$$

Proof. From (4) and (9), we observe that

$$
\begin{aligned}
E i_{k, \lambda}\left(\frac{t}{1-t} x\right) & =\sum_{m=1}^{\infty} \frac{(1)_{m, \lambda}}{(m-1) ! m^{k}}\left(\frac{t}{1-t} x\right)^{m} \\
& =\sum_{m=1}^{\infty} \frac{(1)_{m, \lambda} x^{m}}{(m-1) ! m^{k}} \sum_{j=0}^{m}\left(\begin{array}{c}
m \\
j
\end{array}\right)(-1)^{m-j}\left(\frac{1}{1-t}\right)^{j} \\
& =\sum_{m=1}^{\infty} \sum_{j=0}^{m}\left(\begin{array}{c}
m \\
j
\end{array}\right) \frac{(1)_{m, \lambda}(-1)^{m-j} j ! x^{m}}{(m-1) ! m^{k}} \sum_{n=0}^{\infty}\langle j\rangle_{n} \frac{t^{n}}{n !} \\
& =\sum_{n=0}^{\infty} \sum_{m=1}^{\infty} \sum_{j=0}^{m}\left(\begin{array}{c}
m \\
j
\end{array}\right) \frac{(1)_{m, \lambda}(-1)^{m-j}\langle j\rangle_{n}}{(m-1) ! m^{k}} x^{m} \frac{t^{n}}{n !}
\end{aligned}
$$

Combining (15) and (21), we get

$$
\begin{gathered}
\sum_{m=1}^{\infty} \sum_{j=0}^{m}\left(\begin{array}{c}
m \\
j
\end{array}\right) \frac{(1)_{m, \lambda}(-1)^{m-j}}{(m-1) ! m^{k}} x^{m}=0, \\
L b_{n, \lambda}^{(k)}(x)=\sum_{d=1}^{\infty} \sum_{j=0}^{d}\left(\begin{array}{l}
d \\
j
\end{array}\right) \frac{(1)_{d, \lambda}(-1)^{d-j}\langle j\rangle_{n}}{(d-1) ! d^{k}} x^{d}, \quad n \geq 1 .
\end{gathered}
$$

Theorem 3. For $k \in \mathbb{Z}$ and $n \geq 1$, we have

$$
L b_{n, \lambda}^{(k)}(x)=\sum_{j=1}^{n} \sum_{m=1}^{j} L(n, j) S_{1, \lambda}(j, m) b e l_{m, \lambda}^{(k)}(x),
$$

where bel $l_{m, \lambda}^{(k)}(x)$ are poly-Bell polynomials.

Proof. Replacing $t$ by $\log _{\lambda}(1 /(1-t))$ in (14), we obtain

$$
\begin{aligned}
1+E i_{k, \lambda}\left(\frac{t}{1-t} x\right) & =1+\sum_{m=1}^{\infty} b e l_{m, \lambda}^{(k)} \frac{1}{m !}\left(\log _{\lambda}\left(\frac{1}{1-t}\right)\right)^{m} \\
& =1+\sum_{m=1}^{\infty} b e l_{m, \lambda}^{(k)}(x) \frac{1}{m !}\left(\log _{\lambda}\left(1+\frac{t}{1-t}\right)\right)^{m} \\
& =1+\sum_{m=1}^{\infty} b e l_{m, \lambda}^{(k)}(x) \sum_{j=m}^{\infty} S_{1, \lambda}(j, m) \frac{1}{j !}\left(\frac{t}{1-t}\right)^{j} \\
& =1+\sum_{j=1}^{\infty} \sum_{m=1}^{j} b e l_{m, \lambda}^{(k)}(x) S_{1, \lambda}(j, m) \sum_{n=j}^{\infty} L(n, j) \frac{t^{n}}{n !} \\
& =1+\sum_{n=1}^{\infty} \sum_{j=1}^{n} \sum_{m=1}^{j} S_{1, \lambda}(j, m) L(n, j) b e l_{m, \lambda}^{(k)}(x) \frac{t^{n}}{n !} .
\end{aligned}
$$

Combining (15) and (24), we get the desired result.

The next theorem is the inversion formula of Theorem 3.

Theorem 4. For $k \in \mathbb{Z}$ and $n \geq 1$,

$$
b e l_{n, \lambda}^{(k)}(x)=\sum_{j=1}^{n} \sum_{m=1}^{j}\left(\begin{array}{c}
n \\
j
\end{array}\right)(-m)_{n-j, \lambda} S_{2, \lambda}(j, m) L b_{m, \lambda}^{(k)}(x),
$$

where bel $_{m, \lambda}^{(k)}(x)$ are the degenerate poly-Bell polynomials.

Proof. Replacing $t$ by $1-e_{\lambda}^{-1}(t)$ in (15), from (5), we observe that

$$
\begin{aligned}
& E i_{k, \lambda}\left(x\left(e_{\lambda}(t)-1\right)\right)+1=1+\sum_{m=1}^{\infty} L b_{m, \lambda}^{(k)}(x) \frac{\left(1-e_{\lambda}^{-1}(t)\right)^{m}}{m !} \\
& =1+\sum_{m=1}^{\infty} L b_{m, \lambda}^{(k)}(x) e_{\lambda}^{-m}(t) \frac{\left(e_{\lambda}(t)-1\right)^{m}}{m !} \\
& =1+\sum_{m=1}^{\infty} L b_{m, \lambda}^{(k)}(x) \sum_{h=0}^{\infty}(-m)_{h, \lambda} \frac{t^{h}}{h !} \sum_{j=m}^{\infty} S_{2, \lambda}(j, m) \frac{t^{j}}{j !} \\
& =1+\sum_{j=1}^{\infty} \sum_{m=1}^{j} L b_{m, \lambda}^{(k)}(x) S_{2, \lambda}(j, m) \frac{t^{j}}{j !} \sum_{h=0}^{\infty}(-m)_{h, \lambda} \frac{t^{h}}{h !} \\
& =1+\sum_{n=1}^{\infty} \sum_{j=1}^{n} \sum_{m=1}^{j}\left(\begin{array}{c}
n \\
j
\end{array}\right)(-m)_{n-j, \lambda} S_{2, \lambda}(j, m) L b_{m, \lambda}^{(k)}(x) \frac{t^{n}}{n !} .
\end{aligned}
$$

By (14) and (20), we obtain the desired identity. 
Theorem 5. For $k \in \mathbb{Z}$, the recurrence relation for $L b_{n, \lambda}^{(k)}(x)$ is $L b_{n+1, \lambda}^{(k)}(x)-n L b_{n, \lambda}^{(k)}(x)=\frac{1}{n+1}\left(L b_{n+1, \lambda}^{(k-1)}(x)+L b_{n, \lambda}^{(k-1)}(x)\right)$ $L b_{1, \lambda}^{(k)}(x)=L b_{1, \lambda}^{(k-1)}(x)$.
Proof. Differentiating with respect to $t$ at (15), we observe that

$$
\begin{aligned}
\frac{\partial}{\partial t} E i_{k, \lambda}\left(\frac{t}{1-t} x\right) & =\frac{\partial}{\partial t}\left(\sum_{n=1}^{\infty} \frac{(1)_{n, \lambda}(t /(1-t) x)^{n}}{(n-1) ! n^{k}}\right) \\
& =\frac{x}{(1-t)^{2}}\left(\sum_{n=0}^{\infty} \frac{(1)_{n, \lambda}(t /(1-t) x)^{n-1}}{(n-1) ! n^{k-1}}\right) \\
& =\frac{1}{t(1-t)}\left(\sum_{n=1}^{\infty} \frac{(1)_{n, \lambda}(t /(1-t) x)^{n}}{(n-1) ! n^{k-1}}\right) \\
& =\frac{1}{t} E i_{k-1, \lambda}\left(\frac{t}{1-t} x\right)+\frac{1}{1-t} E i_{k-1, \lambda}\left(\frac{t}{1-t} x\right) \\
& =\sum_{n=0}^{\infty} \frac{L b_{n+1, \lambda}^{(k-1)}}{n+1} \frac{t^{n}}{n !}+\frac{1}{1-t} \sum_{n=1}^{\infty} L b_{n, \lambda}^{(k-1)}(x) \frac{t^{n}}{n !} .
\end{aligned}
$$

On the other hand, from (15), we get

From (28) and (29), we get

$$
\begin{aligned}
\frac{\partial}{\partial t} E i_{k, \lambda}\left(\frac{t}{1-t} x\right) & =\frac{\partial}{\partial t}\left(\sum_{n=1}^{\infty} L b_{n, \lambda}^{(k)}(x) \frac{t^{n}}{n !}\right) \\
& =\sum_{n=1}^{\infty} L b_{n, \lambda}^{(k)}(x) \frac{t^{n-1}}{(n-1) !}=\sum_{n=0}^{\infty} L b_{n+1, \lambda}^{(k)}(x) \frac{t^{n}}{n !}
\end{aligned}
$$

$$
(1-t) \sum_{n=0}^{\infty} L b_{n+1, \lambda}^{(k)}(x) \frac{t^{n}}{n !}=(1-t) \sum_{n=0}^{\infty} \frac{1}{n+1} L b_{n+1, \lambda}^{(k-1)}(x) \frac{t^{n}}{n !}+\sum_{n=1}^{\infty} L b_{n, \lambda}^{(k-1)}(x) \frac{t^{n}}{n !}
$$

From (30), we have

$$
\sum_{n=0}^{\infty} L b_{n+1, \lambda}^{(k)}(x) \frac{t^{n}}{n !}-\sum_{n=0}^{\infty} L b_{n+1, \lambda}^{(k)}(x) \frac{t^{n+1}}{n !}=\sum_{n=0}^{\infty} \frac{1}{n+1} L b_{n+1, \lambda}^{(k-1)}(x) \frac{t^{n}}{n !}-\sum_{n=0}^{\infty} \frac{1}{n+1} L b_{n+1, \lambda}^{(k-1)}(x) \frac{t^{n+1}}{n !}+\sum_{n=1}^{\infty} L b_{n, \lambda}^{(k-1)}(x) \frac{t^{n}}{n !}
$$

From (31), we have

$$
\sum_{n=0}^{\infty} L b_{n+1, \lambda}^{(k)}(x) \frac{t^{n}}{n !}-\sum_{n=1}^{\infty} n L b_{n, \lambda}^{(k)}(x) \frac{t^{n}}{n !}=\sum_{n=0}^{\infty} \frac{1}{n+1} L b_{n+1, \lambda}^{(k-1)}(x) \frac{t^{n}}{n !}-\sum_{n=1}^{\infty} \frac{n}{n+1} L b_{n, \lambda}^{(k-1)}(x) \frac{t^{n}}{n !}+\sum_{n=1}^{\infty} L b_{n, \lambda}^{(k-1)}(x) \frac{t^{n}}{n !}
$$


By comparing the coefficients of both sides of (32), we get the desired result.

$$
\begin{aligned}
\frac{\partial}{\partial x} E i_{k}\left(\frac{t}{1-t} x\right) & =\frac{\partial}{\partial x} \sum_{n=1}^{\infty} \frac{(1)_{n, \lambda}((t /(1-t)) x)^{n}}{(n-1) ! n^{k}} \\
& =\frac{t}{1-t} \sum_{n=1}^{\infty} \frac{(1)_{n, \lambda}((t /(1-t)) x)^{n-1}}{(n-1) ! n^{k-1}} \\
& =\frac{t}{1-t}\left(\frac{t}{1-t} x\right)^{-1} \sum_{n=1}^{\infty} \frac{(1)_{n, \lambda}((t /(1-t)) x)^{n}}{(n-1) ! n^{k-1}}=\frac{1}{x} E i_{k-1, \lambda}\left(\frac{t}{1-t} x\right)
\end{aligned}
$$

Therefore, for $k \geq 0$, by (33), we have

$$
\begin{aligned}
& E i_{k, \lambda}\left(\frac{t}{1-t} x\right)=\int_{0}^{x} \frac{1}{y} \int_{0}^{y} \underbrace{\frac{1}{y} \cdots \int_{0}^{y} \frac{1}{y}}_{(k-2) \text {-times }} E i_{1, \lambda}\left(\frac{t}{1-t} y\right) d y \cdots d y \\
& =\int_{0}^{x} \frac{1}{y} \int_{0}^{y} \underbrace{\frac{1}{y} \cdots \int_{0}^{y} \frac{1}{y}}_{(k-2) \text {-times }}\left(e_{\lambda}\left(\frac{t}{1-t} y\right)-1\right) d y \cdots d y \\
& =\int_{0}^{x} \frac{1}{y} \int_{0}^{y} \underbrace{\frac{1}{y} \cdots \int_{0}^{y} \frac{1}{y}}_{(k-2) \text {-times }}\left(\sum_{s=1}^{\infty} \frac{(1)_{s, \lambda}}{s !}\left(\frac{t}{1-t}\right)^{s} y^{s}\right) d y \cdots d y \\
& =\sum_{s=1}^{\infty}(1)_{s, \lambda} \frac{1}{s !}\left(\frac{t}{1-t}\right)^{s} \frac{1}{s^{k-1}} x^{s}=\sum_{n=1}^{\infty} \sum_{s=1}^{n}(1)_{s, \lambda} \frac{1}{s^{k-1}} L(n, s) x^{s} .
\end{aligned}
$$

From (34), we have the same identity of Theorem 1. Proof. From (9) and (13), we obtain

Theorem 6. For $k \in \mathbb{Z}$ and $n \geq 1$,

$L b_{n, \lambda}^{(k)}(x)=\sum_{j=1}^{n} \sum_{\nu=1}^{j} \sum_{h=1}^{v} \frac{(1)_{h, \lambda}}{\nu^{k-1}} S_{1, \lambda}(\nu, h) S_{2, \lambda}(j, v) L(n, j) x^{j}$.

$$
\begin{aligned}
E i_{k, \lambda}\left(\log _{\lambda}(1+t)\right) & =\sum_{\nu=1}^{\infty} \frac{(1)_{\nu, \lambda}\left(\log _{\lambda}(1+t)\right)^{\nu}}{(\nu-1) ! \nu^{k}} \\
& =\sum_{\nu=1}^{\infty} \frac{(1)_{\nu, \lambda}}{v^{k-1}} \frac{1}{\nu !}\left(\log _{\lambda}(1+t)\right)^{\nu}=\sum_{n=1}^{\infty}\left(\sum_{\nu=1}^{n} \frac{(1)_{\nu, \lambda} S_{1, \lambda}(n, \nu)}{\nu^{k-1}}\right) \frac{t^{n}}{n !} .
\end{aligned}
$$

Replacing $t$ by $e_{\lambda}((t /(1-t)) x)-1$ in (36), from (2) and (12), we get 


$$
\begin{aligned}
E i_{k, \lambda}\left(\frac{t}{1-t} x\right) & =\sum_{\nu=1}^{\infty}\left(\sum_{h=1}^{v} \frac{(1)_{h, \lambda} S_{1, \lambda}(\nu, h)}{h^{k-1}}\right) \frac{1}{\nu !}\left(e_{\lambda}\left(\frac{t}{1-t} x\right)-1\right)^{\nu} \\
& =\sum_{\nu=1}^{\infty}\left(\sum_{h=1}^{v} \frac{(1)_{h, \lambda} S_{1, \lambda}(\nu, h)}{h^{k-1}}\right) \sum_{j=v}^{\infty} x^{j} S_{2, \lambda}(j, v) \frac{1}{j !}\left(\frac{t}{1-t}\right)^{j} \\
& =\sum_{j=1}^{\infty} \sum_{\nu=1}^{j}\left(\sum_{h=1}^{v} \frac{(1)_{h, \lambda} S_{1, \lambda}(\nu, h)}{h^{k-1}}\right) S_{2, \lambda}(j, \nu) x^{j} \sum_{n=j}^{\infty} L(n, j) \frac{t^{n}}{n !} \\
& =\sum_{n=1}^{\infty}\left(\sum_{j=1}^{n} \sum_{\nu=1}^{j} \sum_{h=1}^{v} \frac{(1)_{h, \lambda} S_{1, \lambda}(\nu, h)^{k-1}}{\nu^{k-\lambda}} S_{2, \lambda}(j, \nu) L(n, j) x^{j}\right) \frac{t^{n}}{n !} .
\end{aligned}
$$

Combining (15) and (37), we have

$$
\sum_{n=1}^{\infty} L b_{n, \lambda}^{(k)}(x) \frac{t^{n}}{n !}=\sum_{n=1}^{\infty} \sum_{j=1}^{n} \sum_{\nu=1}^{j} \sum_{h=1}^{v} \frac{(1)_{h, \lambda}}{v^{k-1}} S_{1, \lambda}(\nu, h) S_{2, \lambda}(j, v) L(n, j) x^{j} \frac{t^{n}}{n !} .
$$

By comparing the coefficients on both sides of (38), the desired identity is obtained.

Theorem 7. For $k \in \mathbb{Z}$ and $n \geq 1$,

$L b_{n, \lambda}^{(k)}(x)=\sum_{\omega=1}^{n} \sum_{h=1}^{\omega}\left(\beta_{h, \lambda}^{(k)}(1)-\beta_{h, \lambda}^{(k)}\right) S_{2, \lambda}(\omega, h) L(n, \omega) x^{\omega}$,

where $\beta_{n, \lambda}^{(k)}(x)$ and $\beta_{n, \lambda}^{(k)}$ are the degenerate poly-Bernoulli polynomials and numbers, respectively.

Proof. By (5) and (10), we obtain easily

$$
\beta_{n, \lambda}^{(k)}(x)=\sum_{v=0}^{n}\left(\begin{array}{l}
n \\
l
\end{array}\right)(x)_{v, \lambda} \beta_{n-v, \lambda}^{(k)} .
$$

Then, by (40), we obtain

$$
\begin{aligned}
E i_{k, \lambda}\left(\log _{\lambda}(1+t)\right) & =\left(e_{\lambda}(t)-1\right)\left(\sum_{j=0}^{\infty} \beta_{j, \lambda}^{(k)} \frac{t^{j}}{j !}\right) \\
& =\left(\sum_{d=0}^{\infty}(1)_{d, \lambda} \frac{t^{d}}{d !}-1\right)\left(\sum_{j=0}^{\infty} \beta_{j, \lambda}^{(k)} \frac{t^{j}}{j !}\right) \\
& =\sum_{n=0}^{\infty}\left(\sum_{d=0}^{n}(1)_{d, \lambda} \beta_{n-d, \lambda}^{(k)}\right) \frac{t^{n}}{n !}-\sum_{n=0}^{\infty} \beta_{n, \lambda}^{(k)} \frac{t^{n}}{n !} \\
& =\sum_{n=0}^{\infty}\left(\beta_{n, \lambda}^{(k)}(1)-\beta_{n, \lambda}^{(k)}\right) \frac{t^{n}}{n !} .
\end{aligned}
$$

By replacing $t$ with $e_{\lambda}((t /(1-t)) x)-1$ in (41), we get

$$
\begin{aligned}
E i_{k, \lambda}\left(\frac{t}{1-t} x\right) & =\sum_{h=1}^{\infty}\left(\beta_{h, \lambda}^{(k)}(1)-\beta_{h, \lambda}^{(k)}\right) \frac{\left(e_{\lambda}(t /(1-t) x)-1\right)^{h}}{h !} \\
& =\sum_{h=1}^{\infty}\left(\beta_{h, \lambda}^{(k)}(1)-\beta_{h, \lambda}^{(k)}\right) \sum_{\omega=h}^{\infty} S_{2, \lambda}(\omega, h) \frac{1}{\omega !}\left(\frac{t}{1-t} x\right)^{\omega} \\
& =\sum_{j=1}^{\infty} \sum_{h=1}^{\omega}\left(\beta_{h, \lambda}^{(k)}(1)-\beta_{h, \lambda}^{(k)}\right) S_{2, \lambda}(\omega, h) x^{\omega} \sum_{n=\omega}^{\infty} L(n, \omega) \frac{t^{n}}{n !} \\
& =\sum_{n=1}^{\infty}\left(\sum_{\omega=1}^{n} \sum_{h=1}^{\omega}\left(\beta_{h, \lambda}^{(k)}(1)-\beta_{h, \lambda}^{(k)}\right) S_{2, \lambda}(\omega, h) L(n, \omega) x^{\omega}\right) \frac{t^{n}}{n !}
\end{aligned}
$$

Combining (15) and (42), we have 


$$
\sum_{n=1}^{\infty} L b_{n, \lambda}^{(k)}(x) \frac{t^{n}}{n !}=\sum_{n=1}^{\infty}\left(\sum_{\omega=1}^{n} \sum_{h=1}^{\omega}\left(\beta_{h, \lambda}^{(k)}(1)-\beta_{h, \lambda}^{(k)}\right) S_{2, \lambda}(\omega, h) L(n, \omega) x^{\omega}\right) \frac{t^{n}}{n !} .
$$

By comparing the coefficients on both sides of (43), we attain the desired result.

The next theorem is the inversion formula of Theorem 7.
Theorem 8. For $k \in \mathbb{Z}$, we have

$$
\beta_{n, \lambda}^{(k)}=\sum_{\nu=0}^{n} \sum_{m=h}^{v+1} \sum_{h=1}^{m}\left(\begin{array}{l}
n \\
v
\end{array}\right) \frac{1}{v+1} L(m, h) S_{1, \lambda}(v+1, m) L b_{h, \lambda}^{(k)} \beta_{n-v, \lambda}
$$

where $\beta_{n, \lambda}^{(k)}$ are the degenerate poly-Bernoulli numbers.

Proof. Replacing $t$ by $1-\left(1 /\left(1+\log _{\lambda}(1+t)\right)\right)$ in (15), from (2) and (13), we get

$$
\begin{aligned}
E i_{k, \lambda}\left(\log _{\lambda}(1+t)\right) & =\sum_{h=1}^{\infty} L b_{h, \lambda}^{(k)} \frac{1}{h !}\left(1-\frac{1}{\log _{\lambda}(1+t)+1}\right)^{h} \\
& =\sum_{h=1}^{\infty} L b_{h, \lambda}^{(k)} \sum_{m=h}^{\infty} L(m, h) \frac{1}{m !}\left(\log _{\lambda}(1+t)\right)^{m} \\
& =\sum_{m=1}^{\infty}\left(\sum_{h=1}^{m} L b_{h, \lambda}^{(k)} L(m, h)\right) \sum_{n=m}^{\infty} S_{1, \lambda}(n, m) \frac{t^{n}}{n !} \\
& =\sum_{n=1}^{\infty}\left(\sum_{m=1}^{n} \sum_{h=1}^{m} L b_{h, \lambda}^{(k)} L(m, h) S_{1, \lambda}(n, m)\right) \frac{t^{n}}{n !} .
\end{aligned}
$$

From (10) and (45), we get

$$
\begin{aligned}
\sum_{n=0}^{\infty} \beta_{n, \lambda}^{(k)} \frac{t^{n}}{n !} & =\frac{t}{e_{\lambda}(t)-1} \frac{1}{t} E i_{k, \lambda}\left(\log _{\lambda}(1+t)\right) \\
& =\sum_{i=0}^{\infty} \beta_{i, \lambda} \frac{t^{i}}{i !}\left(\sum_{\nu=0}^{\infty} \sum_{m=1}^{v+1} \sum_{h=1}^{m} \frac{L b_{h, \lambda}^{(k)} L(m, h) S_{1, \lambda}(\nu+1, m)}{j+1}\right) \frac{t^{\nu}}{\nu !} \\
& =\sum_{n=0}^{\infty}\left(\sum_{v=0}^{n} \sum_{m=h}^{v+1} \sum_{h=1}^{m}\left(\begin{array}{l}
n \\
v
\end{array}\right) \frac{1}{v+1} L(m, h) S_{1, \lambda}(v+1, m) L b_{h, \lambda}^{(k)} \beta_{n-v, \lambda}\right) \frac{t^{n}}{n !}
\end{aligned}
$$

By comparing with the coefficients on both sides of (46), we attain the desired result.

Theorem 9. For $k \in \mathbb{Z}$ and $n \geq 1$, we have 


$$
L b_{n, \lambda}^{(k)}(x)=\frac{1}{2} \sum_{j=1}^{n} \sum_{l=1}^{j}\left(\sum_{w=1}^{l}\left(\begin{array}{l}
l \\
w
\end{array}\right)(1)_{w, \lambda} \mathbb{G}_{l-w, \lambda}^{(k)}+2 \mathbb{G}_{l, \lambda}^{(k)}\right) S_{2, \lambda}(j, l) L(n, j) x^{j}
$$

where $\mathbb{G}_{n, \lambda}^{(k)}$ are called the degenerate poly-Genocchi numbers. $\quad$ Proof. From $(11)$ and $\mathbb{G}_{0, \lambda}^{(k)}=0$, we observe that

$$
\begin{aligned}
2 E i_{k, \lambda}\left(\log _{\lambda}(1+t)\right) & =\left(e_{\lambda}(t)+1\right)\left(\sum_{i=0}^{\infty} \mathbb{G}_{i, \lambda}^{(k)} \frac{t^{i}}{i !}\right) \\
& =\left(\sum_{w=0}^{\infty}(1)_{w, \lambda} \frac{t^{w}}{w !}\right)\left(\sum_{i=0}^{\infty} \mathbb{G}_{i, \lambda}^{(k)} \frac{t^{i}}{i !}\right)+\sum_{i=1}^{\infty} \mathbb{G}_{i, \lambda}^{(k)} \frac{t^{i}}{i !} \\
& =\left(\sum_{w=1}^{\infty}(1)_{w, \lambda} \frac{t^{w}}{w !}\right)\left(\sum_{i=0}^{\infty} \mathbb{G}_{i, \lambda}^{(k)} \frac{t^{i}}{i !}\right)+2 \sum_{i=1}^{\infty} \mathbb{G}_{i, \lambda}^{(k)} \frac{t^{i}}{i !} \\
& =\sum_{l=1}^{\infty}\left(\sum_{w=1}^{l}\left(\begin{array}{c}
l \\
w
\end{array}\right)(1)_{w, \lambda} \mathbb{G}_{l-w, \lambda}^{(k)}(1)+2 \mathbb{G}_{l, \lambda}^{(k)}\right) \frac{t^{l}}{l !} .
\end{aligned}
$$

On the one hand, replacing $t$ by $e_{\lambda}((t /(1-t)) x)-1$ in (48), from (2) and (12), we get

$$
\begin{aligned}
2 E i_{k, \lambda}\left(\frac{t}{1-t} x\right) & =\sum_{l=1}^{\infty}\left(\sum_{w=1}^{l}\left(\begin{array}{c}
l \\
w
\end{array}\right)(1)_{w, \lambda} \mathbb{G}_{l-w, \lambda}^{(k)}+2 \mathbb{G}_{l, \lambda}^{(k)}\right) \frac{1}{l !}\left(e_{\lambda}\left(\frac{t}{1-t} x\right)-1\right)^{l} \\
& =\sum_{l=1}^{\infty}\left(\sum_{w=1}^{l}\left(\begin{array}{c}
l \\
w
\end{array}\right)(1)_{w, \lambda} \mathbb{G}_{l-w, \lambda}^{(k)}+2 \mathbb{G}_{l, \lambda}^{(k)}\right) \sum_{j=l}^{\infty} S_{2, \lambda}(j, l) \frac{1}{j !}\left(\frac{t}{1-t}\right)^{j} x^{j} \\
& =\sum_{n=1}^{\infty} \sum_{j=1}^{n} \sum_{l=1}^{j}\left(\sum_{w=1}^{l}\left(\begin{array}{c}
l \\
w
\end{array}\right)(1)_{w, \lambda} \mathbb{G}_{l-w, \lambda}^{(k)}+2 \mathbb{G}_{l, \lambda}^{(k)}\right) S_{2, \lambda}(j, l) L(n, j) x^{j} \frac{t^{n}}{n !} .
\end{aligned}
$$

On the other hand, by (15) and (49), we have

$$
\begin{aligned}
\sum_{n=1}^{\infty} L b_{k, n, \lambda}(x) \frac{t^{n}}{n !} & =E i_{k, \lambda}\left(\frac{t}{1-t} x\right) \\
& =\frac{1}{2} \sum_{n=1}^{\infty} \sum_{j=1}^{n} \sum_{l=1}^{j}\left(\sum_{w=1}^{l}\left(\begin{array}{l}
l \\
w
\end{array}\right)(1)_{w, \lambda} \mathbb{G}_{l-w, \lambda}^{(k)}+2 \mathbb{G}_{l, \lambda}^{(k)}\right) S_{2, \lambda}(j, l) L(n, j) x^{j} \frac{t^{n}}{n !}
\end{aligned}
$$

By comparing the coefficients on both sides of (50), we get the desired identity. 


\section{Conclusion}

In this paper, we considered the degenerate poly-Lah-Bell polynomials and numbers by using of the degenerate polyexponential functions and obtained some combinatorial identities between these polynomials and numbers and special polynomials and numbers, involving the explicit formula, Dobinski-like formula, and recurrence relation. In addition, we derived the relations between these polynomials and the degenerate poly-Bell polynomials which we recently introduced [4]. Finally, we would like to continue researching "poly" about the generalized Stirling polynomials and numbers.

\section{Data Availability}

No data were used to support this study.

\section{Ethical Approval}

All authors reveal that there is no ethical problem in the production of this paper.

\section{Disclosure}

After this paper was submitted, the authors knew from the editor that the following paper is related to this paper. Therefore, the authors inform to the following paper for the readers. T. Kim, D.S. Kim, H.Y. Kim, and J. Kwon introduced A New Type Degenerate Daehee Numbers and Polynomials, arxiv.org/abs/2004.08743a

\section{Conflicts of Interest}

The authors declare that there are no conflicts of interest regarding the publication of this article.

\section{Authors' Contributions}

TK and HKK conceived the framework and structured the whole paper. HKK wrote the whole paper. TK and HKK checked the results of the paper and completed the revision of the article.

\section{Acknowledgments}

This work was supported by the Basic Science Research Program, the National Research Foundation of Korea (NRF2021R1F1A1050151).

\section{References}

[1] S. Araci and Y. Hamahata, "Degenerate poly-type 2-Bernoulli polynomials," Mathematical Sciences and Applications, vol. 9, no. 1, pp. 1-8, 2012.

[2] A. Bayad and Y. Hamahata, "Polylogarithms and poly-Bernoulli polynomials," Kyushu Journal of Mathematics, vol. 65, no. 1, pp. 15-24, 2012.

[3] T. Kim, D. S. Kim, J. K. Kwon, and H. Y. Kim, "A note on degenerate Genocchi and poly-Genocchi numbers and polynomials," Journal of Inequalities and Applications, vol. 110, 2020.

[4] T. Kim and H. K. Kim, "Degenerate poly-Bell polynomials and numbers," Advances in Difference Equations, vol. 361, no. 1, 2021.

[5] L. Lewin, Polylogarithms and Associated Functions, With a Foreword by A. J. Van der Poorten, North-Holland Publishing, Amsterdam, Netherlands, 1981.

[6] K. Burak, "Degenerate polyexponential functions and polyEuler polynomials," Communications of the Korean Mathematical Society, vol. 36, no. 1, pp. 19-26, 2021.

[7] W. A. Khan, "A note on degenerate Hermite poly-Bernoulli numbers and polynomials," Journal of Classical Analysis, vol. 8, no. 1, pp. 65-76, 2016.

[8] D. S. Kim and T. Kim, "A note on polyexponential amd unipoly functions," Russian Journal of Mathematical Physics, vol. 26, no. 1, pp. 40-49, 2019.

[9] K. Burak, "Explicit relations for the modified degenerate Apostol-type polynomials," Journal of Balikesir University Institute of Science and Technology, vol. 20, no. 2, pp. 401-412, 2018.

[10] U. Duran, M. Acikgoz, and S. Araci, "Construction of the type 2 poly-Frobenius-Genocchi polynomials with their certain applications," Advances in Difference Equations, vol. 14, 2020.

[11] L. Carlitz, "Degenerate Stirling, Bernoulli and Eulerian numbers," Utilitas Mathematica, vol. 15, pp. 51-88, 1979.

[12] I. Lah, "A new kind of numbers and its application in the actuarial mathematics," Boletim do Instituto dos Actuários Portugueses, vol. 9, pp. 7-15, 1954.

[13] L. Comtet, Advanced Combinatorics: The Art of Finite and Infinite Expansions, Reidel, Dordrecht, Netherlands, 1974.

[14] D. S. Kim and T. Kim, "Lah-Bell numbers and polynomials," Proceedings of the Jangjeon Mathematical Society, vol. 23, no. 4, pp. 577-586, 2020.

[15] Y. Simsek, "Identities and relations related to combinatorial numbers and polynomials," Proceedings of the Jangjeon Mathematical Society, vol. 20, no. 1, pp. 127-135, 2017.

[16] G. H. Hardy, "On a class of analytic functions," Proceedings of the London Mathematical Society, vol. s2-3, no. 1, pp. 441-460, 1905. 